https://helda.helsinki.fi

\title{
The Associations Between Leisure-Time Physical Activity and Academic Performance : A Twin Study
}

\section{Aaltonen, Sari}

2021-08

Aaltonen , S , Palviainen , T , Rose , R J , Kujala , U M , Kaprio , J \& Silventoinen , K 2021 , '

The Associations Between Leisure-Time Physical Activity and Academic Performance : A

Twin Study ' , Journal of physical activity \& health , vol. 18 , no. 8 , pp. 998-1003 . https://doi.org/10.1123/jpah.2020-

http://hdl.handle.net/10138/337638

https://doi.org/10.1123/jpah.2020-0746

unspecified

acceptedVersion

Downloaded from Helda, University of Helsinki institutional repository.

This is an electronic reprint of the original article.

This reprint may differ from the original in pagination and typographic detail.

Please cite the original version. 
1 The Associations between Leisure-Time Physical Activity and Academic

2 Performance: A Twin Study

3

4 ABSTRACT

5 Background Both genetic and environmental influences have been shown to contribute to

6 the association between physical activity and overall academic performance. We examined

7 whether leisure-time physical activity (LTPA) shares genetic and environmental variances

8 between spelling, essay writing, reading aloud, reading comprehension and mathematics in

9 early adolescence. Moreover, we investigated whether genetic polymorphisms associated

10 with physical activity behavior affect these academic skills.

11 Methods Participants were 12-year-old Finnish twins ( $\mathrm{n}=4356-4370$ twins/academic skill,

$1249 \%$ girls). Academic skills were assessed by teachers and LTPA was self-reported. Polygen-

13 ic scores for physical activity behavior were constructed from the UK Biobank. Quantitative

14 genetic modeling and linear regression models were used to analyze the data.

15 Results The trait correlations between LTPA and academic skills were significant but weak

$16(\mathrm{r}=0.05-0.08)$. The highest trait correlation was found between LTPA and mathematics. A

17 significant genetic correlation was revealed between LTPA and essay writing $\left(\mathrm{r}_{\mathrm{A}}=0.14\right)$. Re-

18 garding polygenic scores of physical activity, the highest correlations were found with read-

19 ing comprehension, spelling and essay writing, but these results only approached statistical

20 significance (p-values 0.09-0.15).

21 Conclusions Our results suggest that reading and writing are the academic skills that most

22 likely share a common genetic background with LTPA. 
23 There is a strong body of research on the association between physical activity and academic

24 performance ${ }^{1-4}$. However, recent systematic reviews have concluded that the levels of associations between physical activity and different academic skills vary greatly ${ }^{5,6}$. The strongest evidence for the association with physical activity was found for mathematics by Singh et al. $(2019)^{5}$, while Haverkamp et al. $(2020)^{6}$ only demonstrated a significant effect of physical activity on academic skills within the language domain. In addition to these conflicting association findings within different academic skills, the direction of the potential association and the nature of causality between physical activity and academic performance has also remained under debate without any clear results on whether the association constitutes a causal effect $^{7-10}$.

Previous studies have also shown that similar to overall academic performance, mathematicand reading-specific academic skills are highly heritable ${ }^{11,12}$. Moreover, physical activity has been shown to be moderately heritable ${ }^{13,14}$. We have also shown, contrary to the idea of causality, that the association between leisure-time physical activity (LTPA) and grade point average can partly reflect overlapping genetic and familial influences ${ }^{7}$. Therefore, a better understanding of common genetic and familial background that potentially account for the associations between physical activity and different academic skills would be warranted.

In this study, we aimed to examine to what extent LTPA and academic performance in spelling, essay writing, reading aloud, reading comprehension and mathematics share genetic and environmental influences in early adolescence using genetic twin modeling and polygenic scores (PGS). By using these two different methods, each making different methodological assumptions, we are able to analyze the genetic background of LTPA and academic performance more comprehensively than when relying on only one method. 
METHODS

The participants of this study were drawn from the FinnTwin12 study, which is a populationbased longitudinal study of health and behavior in Finnish twins born in 1983-1987 ${ }^{15}$. The twins and their parents completed study questionnaires on health, behavior, lifestyle and social/interpersonal environments when the twins were 11-12 years old (age range 10.8-12.3 years). The response rate was $90 \%$. Most of the twins were in the same class and had the same teacher who usually had a long-term teaching relationship with the twins. The teachers assessed the twins' behavior and academic skills at school. In this study, we had data available on LTPA and academic skills from 4356 to 4370 twins per skill (51\% boys and $49 \%$ girls) including 2102 full twin pairs. DNA was extracted from blood and saliva samples collected when the twins were young adults (mean age 24.4 years). Within the sample, $32 \%$ of the twins were monozygotic, while $31 \%$ were same-sex dizygotic and $31 \%$ were opposite-sex dizygotic twins.

61 ASSESSMENT OF ACADEMIC PERFORMANCE

The teachers of the twins assessed spelling, essay writing, reading aloud, reading comprehension and mathematics when the twins were at the mean age of 11.4 years with the following question specifically tailored for the study: "Please, evaluate a twin's performance in the following academic skills compared to the average pupil in your class?". The response options were categorized as follows: 1) clearly below the average, 2) slightly below the average, 3) average, 4) slightly above the average, and 5) clearly above the average.

69 


\section{ASSESSMENT OF LEISURE-TIME PHYSICAL ACTIVITY}

Twins self-reported their LTPA based on a structured question on the frequency of LTPA excluding physical education classes at school: "How often do you exercise in your leisure time?". There were five response options: 1) not at all 2) two to three times in six months, 3) two to three times a month, 4) two to three times a week, and 5) just about every day.

\section{DEMOGRAPHIC VARIABLES}

The age of the twins was calculated based on their date of birth obtained from the Finnish Population Register Centre and the date of return of the study questionnaire. The sex of the twins was also provided by the Finnish Population Register Centre and cross-checked with the self-reported questionnaire data. The zygosity of the twins was mainly based on measured genotypes. However, there were a few twins who did not have a DNA sample and their zygosity was based on questions on physical similarity at age 11-12. This method has been shown to have high validity in this twin cohort $^{16}$.

\section{POLYGENIC SCORES}

Genome-wide genotype data were used to produce PGS for physical activity behavior. The PGSs were constructed for self-reported and accelerometer-measured physical activity from the UK Biobank data that is based on the general UK population between ages $40-69^{17,18}$. The self-reported questions based on the "number of days/week of walked 10+ minutes"

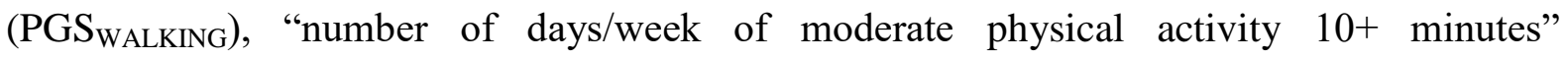

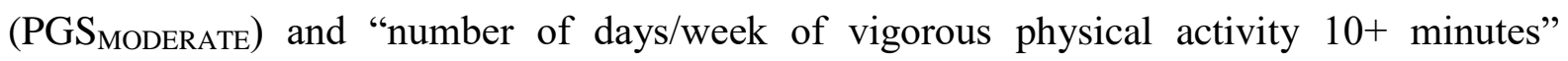

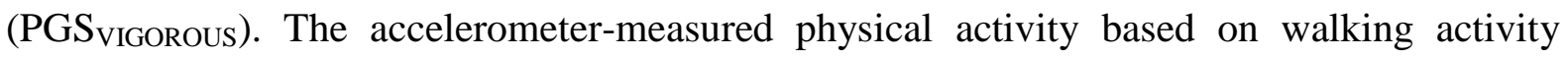
(PGS MEASURED WALKING), moderate intensity activity (PGS MEASURED MODERATE) and overall

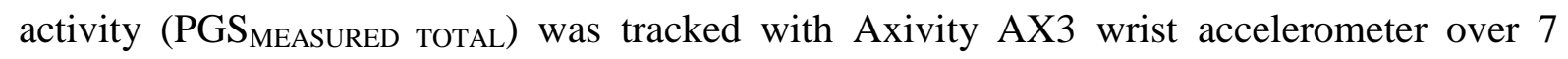


96 days $^{19}$. Kujala et al. $(2020)^{20}$ have reported the details of the genotyping and polygenic scor97 ing.

\section{STATISTICAL METHODS}

100 First, we estimated intra-class correlation coefficients to quantify the degrees to which 101 monozygotic and dizygotic twins resemble each other for LTPA and academic skills (Supplementary table 1). The genetic twin modeling began by decomposing the trait variation in LTPA and academic skills into three components (additive genetic variation (A), shared environmental variation $(\mathrm{C})$, and unique environmental variation $(\mathrm{E})$ ) and comparing different univariate models to select the best-fitting model (Supplementary table 2$)^{21}$. Based on the best-fitting univariate model, we estimated genetic and environmental contributions to LTPA and academic skills by sex (Supplementary table 3): the contributions of genetic influences to LTPA were $30 \%$ in boys and $17 \%$ in girls, whereas the heritability estimates for the academic skills ranged from $64 \%$ to $77 \%$ in boys and from $53 \%$ to $69 \%$ in girls. Next, bivariate

110 Cholesky decompositions were conducted to estimate trait correlations between LTPA and 111 academic skills ${ }^{22}$. We further decomposed these trait correlations into genetic and environmental correlations and estimated to what extent the proportions of the trait correlations are explained by genetic and environmental factors. The bivariate Cholesky decompositions were

114 also used to derive a test of causality between these two traits: a causal association should 115 appear as both genetic and environmental correlations ${ }^{23}$. The correlations were initially per-

116 formed based on the univariate model-fitting results. However, decompositions for boys and 117 girls separately could not be reliably estimated; thus, we present the bivariate Cholesky decomposition results for both sexes as main findings. The findings for boys and girls separate-

119 ly are shown in Supplementary table 4. OpenMx software (version 2.0.1) was used for these 120 quantitative genetic analyses ${ }^{24}$. 
122 We used linear regression models to analyze whether the academic skills were associated

123 with genetic susceptibility to physical activity behavior (presented as PGSs). The physical

124 activity-related PGSs and academic skills were scaled to obtain standardized normal distribu-

125 tion with a mean of 0 and standard deviation of 1 . The regression models were adjusted for

126 sex and the first 10 genetic principal components to control for population stratification. Be-

127 cause we analyzed twins as individuals, the regression models were controlled for the cluster-

128 ing of twins within pairs because the observations between co-twins may be correlated. We

129 used Stata 14.1 software (StataCorp, College Station, Texas, USA) to produce linear regres-

130 sion models as well as baseline statistics. Descriptive statistics are presented in Supplemen-

131 tary table 5. The means and standard deviations of LTPA and academic skills stratified by 132 zygosity and sex are provided in Supplementary table 6.

\section{ETHICS OF THE STUDY}

135 The ethics committee of the Department of Public Health of the University of Helsinki (Fin-

136 land), the ethics committee of the Helsinki University Central Hospital District (Finland) and

137 the Institutional Review Board of Indiana University (USA) approved the FinnTwin12 study

138 protocol. The parents of the twins initially provided written informed consent for study par-

139 ticipation, but as young adults, the twins themselves provided written informed consent for

140 genetic analyses.

142 RESULTS

143 The trait correlations between LTPA and academic skills were positive and statistically sig-

144 nificant but weak (from 0.05 to 0.08 ) (Table 1). The highest trait correlation was found be-

145 tween LTPA and mathematics. Based on the Cholesky decomposition, common genetic in- 
146 fluences statistically significantly contributed to the association between LTPA and essay

147 writing $\left(r_{A}=0.14\right)$, supporting a genetically-influenced mechanism underlying the association.

148 The next highest genetic correlations were found between LTPA and reading aloud as well as

149 between LTPA and mathematics (both $\mathrm{r}_{\mathrm{A}}=0.11$ ), but these findings did not reach statistical

150 significance. Even though the importance of familial factors in explaining the associations

151 between LTPA and academic skills was highlighted by intra-class correlation coefficients and

152 by the fact that shared environmental influences could not be dropped from the best-fitting

153 final models (Supplementary table 2), no significant shared environmental correlations were

154 found between LTPA and different academic skills. Furthermore, no significant unique envi-

155 ronmental correlations between LTPA and academic skills were found.

157 The associations between genetic susceptibility to physical activity behavior (i.e., PGSs) and academic skills are shown in Table 2 . The analyses revealed weak and non-significant associations between the PGSs for physical activity behavior and academic skills: positive within

160 PGSs based on the accelerometer-measured physical activity and mostly negative within

161 PGSs based on the questionnaire-based physical activity. With regard to accelerometermeasured physical activity, the highest associations were found between PGS $\mathrm{SEASURED} \mathrm{WALK-}_{\text {- }}$ ING and spelling and essay writing (both $\mathrm{r}=0.05$ ), but these results only lean toward statistical significance ( $\mathrm{p}=0.13$ and $\mathrm{p}=0.15$, respectively). Although many associations related to PGSs

165 based on the questionnaire-based physical activity were even lower than those based on the

166 accelerometer-measured physical activity, the association between PGS $S_{\text {VIGORous }}$ and reading 167 comprehension $(\mathrm{r}=-0.06)$ approached statistical significance $(\mathrm{p}=0.09)$. The next highest associations regarding the PGSs based on the questionnaire-based physical activity behavior were

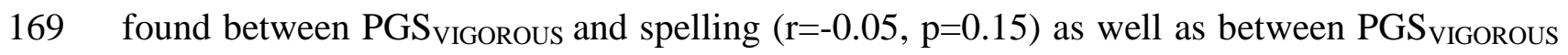
170 and essay writing $(\mathrm{r}=-0.06, \mathrm{p}=0.15)$. 


\section{DISCUSSION}

172 By using genetically informative twin data, we examined the genetic and familial associa-

173 tions between LTPA and academic skills in spelling, essay writing, reading aloud, reading

174 comprehension and mathematics in early adolescence. Regarding the twin modeling, the most

175 apparent finding to emerge was that all academic skills were positively associated with

176 LTPA. We found the highest association between LTPA and mathematics. However, these

177 observed associations between LTPA and academic skills shared genetic influences to a small

178 extent; only the association between LTPA and essay writing was found to have a significant

179 genetic component. Reading aloud and mathematics showed the next highest genetic correla-

180 tion with LTPA, but without statistical significance. We found no significant environmental

181 correlations (neither shared nor unique environmental correlations) between LTPA and aca-

182 demic skills.

183

184 Contrary to the twin modeling results, PGSs for physical activity behavior were not signifi-

185 cantly associated with academic skills - however, the results related to reading comprehen-

186 sion, spelling and essay writing approached a customary level of statistical significance. It is

187 important to note that PGSs were based on age groups older (i.e., 40-69-year-olds) than our

188 study participants (i.e., 11-12-year-olds). This may potentially affect the associations found

189 between PGSs and academic skills. The reality of the associations may be represented more

190 accurately by PGSs based on the accelerometer-measured physical activity than based on the

191 questionnaire-based physical activity because accelerometers may better reflect voluntary

192 physical activity behavior and inherent physical activity abilities of the individual, regardless

193 of the individual's age.

194 
195 This study supports evidence from previous observations indicating a positive association

196 between LTPA and academic performance ${ }^{1-3}$. We found the highest trait correlation between

197 LTPA and mathematics, which reflects the results of the meta-analysis of Singh et al.

$198(2019)^{5}$. Moreover, our study confirms the results of the previous studies indicating that

199 LTPA is moderately ${ }^{13,14}$ and academic performance highly heritable ${ }^{11,12,25,26}$. We found a sta-

200 tistically significant genetic correlation $\left(r_{A}=0.14\right)$ regarding the association between LTPA

201 and essay writing. This result, along with the non-overlapping shared environmental and nonoverlapping unique environmental influences between LTPA and essay writing, challenges the assumption of a potential causal relationship between LTPA and essay writing by indicating that there is a genetic relationship between these two traits (a causal association between LTPA and essay writing should appear as both genetic and environmental correlations). The genetic correlation of 0.14 we found between LTPA and essay writing is also in line with the genetic correlation estimate we found in our previous study between LTPA and grade point average for boys at age $12\left(\mathrm{r}_{\mathrm{A}}=0.17\right)$ when using the same data-set ${ }^{7}$. Overall, our twin modeling and analyses related to PGSs show that reading and writing are the academic skills that

210 most likely share a common genetic background: results are systematic yet not statistically

211 significant regarding all estimates. Speculatively, these common genetic backgrounds might explain the trait correlations found between these traits as well as suggest that reading and

213 writing represent, to a great extent, a grade point average.

215 In light of our previous $\operatorname{study}^{7}$, showing that the association between LTPA and grade point average was also partly explained by the overlapping familial environmental influences, it is

217 somewhat surprising that common familial background was not found to exist between LTPA 218 and any academic skill. It is possible that the academic performance data used in the current 219 study did not reliably estimate shared environmental influences: the confidence intervals of 
220 shared environmental correlations are wide, which may indicate that our data are underpowered to decompose shared environmental influences explaining the proportions of the trait correlations between LTPA and academic skills.

224 We focused on twins' frequency of LTPA, which greatly reflects voluntary behavior. Our assessment may be a restricted picture of the total LTPA, but it still represents twins' physical activity behavior in their leisure time. The validity of physical activity questionnaires used in

227 Finnish twins have been demonstrated ${ }^{27,28}$. Academic skills were reported by teachers. The measurements were not standardized and not totally comparable but teachers evaluated the twins' skills as objectively as possible. In Finland, practically all teachers have undergone

230 Master's level training and schools follow a national curriculum meaning that teachers' evaluations are based on similar principles.

233 A further limitation is that our study design was cross-sectional. Even though our cross-

234 sectional twin data contain genetic information that can be used to derive a test of causality

235 between leisure-time physical activity and academic skills ${ }^{23,29}$, longitudinal studies would be more informative about the genetic and environmental influences behind the long-term associations between LTPA and academic skills. This is because the twin modeling results are always age- and time-specific, as well as sensitive to changes in the overall and environmental variances. For example, we have shown in our previous study that an emotionally warm, supportive, and encouraging family environment in childhood can enhance children's genetic potential for voluntary physical activity even years after the influence of the home environment in childhood ${ }^{30}$. 
244 Major strengths of our study are the population-based sample and its large size and relatively

245 equal sex representation. Due to the very high participation rate, various selection biases are

246 also unlikely in our study. Thus, the generalizability of our study findings is good but limited

247 to individuals at age 11-12 years. A further strength is that we were able to use two different

248 measures to assess genetic influences affecting physical activity behavior: twin modeling and

249 PGSs.

250

251 Despite some limitations, our study certainly adds to the understanding of the association between LTPA and academic performance. The most obvious finding to emerge from this study is that the roots of the associations between LTPA and academic skills related to writing and reading may be due to common genetic influences rather than causality as previously speculated. However, it is important to be cautious interpreting our results because our sample size may not have been large enough for the bivariate twin modeling analyses. Thus, this study should be repeated using larger twin or family samples - this may enable a more reliable assessment of potential overlapping shared environmental influences between LTPA and academic skills.

\section{FUNDING SOURCE}

The research work has been supported by the Academy of Finland (grants 100499, 205585, 141054, 265240, 263278, 264146 and 312073 to J.K.), the Finnish Ministry of Education and Culture (to U.M.K.), the Juho Vainio Foundation (to S.A. and U.M.K.), and the Finnish Cultural Foundation (to S.A.). Data collection of the FinnTwin12 study has been supported by and AA-09203 to R.J.R.). 


\section{AUTHOR CONTRIBUTIONS}

R.J.R. and J.K. designed and contributed to the data collection of the FinnTwin 12 study. S.A., U.M.K., J.K., and K.S. designed the present study. S.A., T.P. and K.S. conducted the statistical analyses. S.A. drafted the manuscript and T.P., R.J.R., J.K., U.M.K. and K.S. critically revised the manuscript. All authors approved the final manuscript. The authors declare no conflict of interest.

\section{REFERENCES}

1. Alvarez-Bueno C, Pesce C, Cavero-Redondo I, Sanchez-Lopez M, Garrido-Miguel M, Martinez-Vizcaino V. Academic achievement and physical activity: A meta-analysis. Pediatrics. 2017;140(6):10.1542/peds.2017-1498. doi: e20171498.

2. Mura G, Vellante M, Nardi AE, Machado S, Carta MG. Effects of school-based physical activity interventions on cognition and academic achievement: A systematic review. CNS Neurol Disord Drug Targets. 2015;14(9):1194-1208.

3. Marques A, Santos DA, Hillman CH, Sardinha LB. How does academic achievement relate to cardiorespiratory fitness, self-reported physical activity and objectively reported physical activity: A systematic review in children and adolescents aged 6-18 years. $\mathrm{Br} J$ Sports Med. 2018;52(16):1039-097361. doi: 10.1136/bjsports-2016-097361.

4. Barbosa A, Whiting S, Simmonds P et al. Physical activity and academic achievement: An umbrella review. Int J Environ Res Public Health. 2020;17(16):10.3390/ijerph17165972. doi: E5972.

5. Singh AS, Saliasi E, van den Berg V, et al. Effects of physical activity interventions on cognitive and academic performance in children and adolescents: A novel combination of a systematic review and recommendations from an expert panel. Br J Sports Med. 2019;53(10): 640-647. 
294 6. Haverkamp BWR, Vertessen K, van Ewijk H, Oosterlaan J, Hartman E. Effects of physical

295 activity interventions on cognitive outcomes and academic performance in adolescents and

296 young adults: A meta-analysis. J Sports Sci. 2020;38(23):2637-2660.

297 7. Aaltonen S, Latvala A, Jelenkovic A, et al. Physical activity and academic performance:

298 Genetic and environmental associations. Med Sci Sports Exerc. 2020;52(2):381-390.

299 8. Lees C, Hopkins J. Effect of aerobic exercise on cognition, academic achievement, and 300 psychosocial function in children: A systematic review of randomized control trials. Prev 301 Chronic Dis. 2013;10:E174. doi: 10.5888/pcd10.130010.

302 9. Aaltonen S, Latvala A, Rose RJ, Kujala UM, Kaprio J, Silventoinen K. Leisure-time 303 physical activity and academic performance: Cross-lagged associations from adolescence to 304 young adulthood. Sci Rep. 2016;6:39215. doi: 10.1038/srep39215.

305 10. Kari JT, Viinikainen J, Bockerman P et al. Education leads to a more physically active 306 lifestyle: Evidence based on Mendelian randomization. Scand J Med Sci Sports.

$307 \quad 2020 ; 30(7): 1194-1204$.

308 11. Kovas Y, Haworth CM, Dale PS, Plomin R. The genetic and environmental origins of 309 learning abilities and disabilities in the early school years. Monogr Soc Res Child Dev. 310 2007;72(3):vii, 1-144. doi: MONO453.

311 12. Davies G, Tenesa A, Payton A, et al. Genome-wide association studies establish that hu312 man intelligence is highly heritable and polygenic. Mol Psychiatry. 2011;16(10):996-1005.

313 13. de Geus EJ, Bartels M, Kaprio J, Lightfoot JT, Thomis M. Genetics of regular exercise 314 and sedentary behaviors. Twin Res Hum Genet. 2014;17(4):262-271.

315 14. Lightfoot JT, DE Geus, E. J. C., Booth FW, et al. Biological/genetic regulation of physi316 cal activity level: Consensus from GenBioPAC. Med Sci Sports Exerc. 2018;50(4):863-873.

317 15. Rose RJ, Salvatore JE, Aaltonen S, et al. FinnTwin12 cohort: An updated review. Twin 318 Res Hum Genet. 2019;22(5):302-311. 

and environmental influences on growth from late childhood to adulthood: A longitudinal study of two Finnish twin cohorts. Am J Hum Biol. 2011;23(6):764-773.

17. Bycroft C, Freeman C, Petkova D, et al. The UK biobank resource with deep phenotyping and genomic data. Nature. 2018;562(7726):203-209.

18. Doherty A, Jackson D, Hammerla N, et al. Large scale population assessment of physical activity using wrist worn accelerometers: The UK biobank study. PLoS One. 2017;12(2):e0169649. doi: 10.1371/journal.pone.0169649.

19. Doherty A, Smith-Byrne K, Ferreira T, et al. GWAS identifies 14 loci for devicemeasured physical activity and sleep duration. Nat Commun. 2018;9(1):5257-4. doi: 10.1038/s41467-018-07743-4.

20. Kujala UM, Palviainen T, Pesonen P, et al. Polygenic risk scores and physical activity. Med Sci Sports Exerc. 2020;52(7):1518-1524.

21. Rijsdijk FV, Sham PC. Analytic approaches to twin data using structural equation models. Brief Bioinform. 2002;3(2):119-133.

22. Posthuma D, Beem AL, de Geus EJ, et al. Theory and practice in quantitative genetics. Twin Res. 2003;6(5):361-376.

23. Verhulst B, Estabrook R. Using genetic information to test causal relationships in crosssectional data. J Theor Polit. 2012;24(3):328-344. and Bates T, Mehta P,Fox J,. OpenMx: An open source extended structural equation modeling framework. Psychometrika. 2011;76(2):306-317. 25. de Zeeuw E.L., de Geus E.J.C., Boomsma D.I. Meta-analysis of twin studies highlights

342 the importance of genetic variation in primary school educational achievement. Trends Neurosci Educ. 2015;4:69-76. 
344 26. Okbay A, Beauchamp JP, Fontana MA, et al. Genome-wide association study identifies

34574 loci associated with educational attainment. Nature. 2016;533(7604):539-542.

346 27. Leskinen T, Waller K, Mutikainen S, et al. Effects of 32-year leisure time physical activ-

347 ity discordance in twin pairs on health (TWINACTIVE study): Aims, design and results for 348 physical fitness. Twin Res Hum Genet. 2009;12(1):108-117.

349 28. Waller K, Kaprio J, Kujala UM. Associations between long-term physical activity, waist

350 circumference and weight gain: A 30-year longitudinal twin study. Int J Obes (Lond). $351 \quad 2008 ; 32(2): 353-361$.

352 29. Duffy DL, Martin NG. Inferring the direction of causation in cross-sectional twin data:

353 Theoretical and empirical considerations. Genetic Epidemiology. 1994;11:483-502.

354 30. Aaltonen S, Kaprio J, Kujala UM, Pulkkinen L, Rose RJ, Silventoinen K. The interplay

355 between genes and psychosocial home environment on physical activity. Med Sci Sports

356 Exerc. 2018;50(4):691-699. 
358 Table 1 Trait correlations $\left(r_{\text {trait }}\right)$ as well as the correlations between additive genetic $\left(r_{A}\right)$, shared environmental $\left(r_{C}\right)$ and unique environmental $\left(r_{E}\right)$

359 influences for LTPA and academic skills with $95 \%$ confidence intervals at age 12.

\begin{tabular}{|c|c|c|c|c|c|c|c|c|c|}
\hline & & & Trait correlation & Additive genetic cor & elation & Shared environmen & al factors & Unique environme & al factors \\
\hline Trait 1 & Trait 2 & Model & $\mathrm{r}_{\text {trait }}(95 \% \mathrm{CI})$ & $\mathrm{r}_{\mathrm{A}}(95 \% \mathrm{CI})$ & $\begin{array}{l}\% \text { Explained } \\
\text { of } \mathrm{r}_{\text {trait }}\end{array}$ & $\mathrm{r}_{\mathrm{C}}(95 \% \mathrm{CI})$ & $\begin{array}{l}\% \text { Explained } \\
\text { of } \mathrm{r}_{\text {trait }}\end{array}$ & $\mathrm{r}_{\mathrm{E}}(95 \% \mathrm{CI})$ & $\begin{array}{l}\% \text { Explained } \\
\text { of } \mathrm{r}_{\text {trait }}\end{array}$ \\
\hline Spelling & LTPA & Sexes combined & 0.05 (0.01 to 0.09$)$ & $0.08(-0.06$ to -0.22$)$ & $80 \%$ & $0.03(-0.35$ to 0.40$)$ & $11 \%$ & $0.02(-0.05$ to 0.09$)$ & $9 \%$ \\
\hline Essay writing & LTPA & Sexes combined & 0.06 (0.02 to 0.09$)$ & $0.14(0.00$ to 0.28$)$ & * & $-0.13(-0.66$ to 0.25$)$ & $*$ & $0.03(-0.05$ to 0.10$)$ & $11 \%$ \\
\hline Reading aloud & LTPA & Sexes combined & 0.05 (0.01 to 0.09$)$ & $0.11(-0.03$ to 0.25$)$ & $*$ & $-0.06(-0.41$ to 0.24$)$ & $*$ & $0.05(-0.02$ to 0.12$)$ & $22 \%$ \\
\hline Reading comprehension & LTPA & Sexes combined & 0.05 (0.01 to 0.09$)$ & $0.01(-0.13$ to 0.14$)$ & $7 \%$ & $0.16(-0.11$ to 0.44$)$ & $78 \%$ & $0.03(-0.04$ to 0.11$)$ & $14 \%$ \\
\hline Mathematics & LTPA & Sexes combined & 0.08 (0.04 to 0.12$)$ & $0.11(-0.04$ to 0.26$)$ & $74 \%$ & $0.06(-0.37$ to 0.46$)$ & $14 \%$ & $0.04(-0.03$ to 0.11$)$ & $12 \%$ \\
\hline
\end{tabular}

CI=confidence intervals, LTPA=leisure-time physical activity, * = cannot be calculated reliably

362 Table 2 Associations between genetic susceptibility to physical activity behavior (presented as polygenic scores) and academic skills when sex

363 and the first 10 principal components are taken into account.

\begin{tabular}{|c|c|c|c|c|c|c|c|c|c|c|c|c|}
\hline & \multicolumn{12}{|c|}{ Genetic susceptibility to physical activity behavior } \\
\hline & \multicolumn{6}{|c|}{ Accelerometer-measured physical activity } & \multicolumn{6}{|c|}{ Questionnaire-based physical activity } \\
\hline & \multicolumn{2}{|c|}{ PGS $_{\text {MEASURED WALKING }}$} & \multicolumn{2}{|c|}{ PGS $_{\text {MEASURED MODERATE }}$} & \multicolumn{2}{|c|}{ PGS $_{\text {MEASURED TOTAL }}$} & \multicolumn{2}{|c|}{ PGS $_{\text {WALKING }}$} & \multicolumn{2}{|c|}{ PGS $_{\text {MODERATE }}$} & \multicolumn{2}{|c|}{ PGS $_{\text {VIGOROUS }}$} \\
\hline & B-coeff. & p-value & B-coeff. & p-value & B-coeff. & p-value & B-coeff. & $\mathrm{p}$-value & B-coeff. & p-value & B-coeff. & p-value \\
\hline Spelling & 0.05 & 0.13 & 0.04 & 0.24 & 0.04 & 0.26 & 0.004 & 0.91 & -0.01 & 0.76 & -0.05 & 0.15 \\
\hline Essay writing & 0.05 & 0.15 & 0.04 & 0.24 & 0.02 & 0.65 & -0.04 & 0.31 & -0.05 & 0.58 & -0.06 & 0.15 \\
\hline Reading aloud & 0.03 & 0.32 & 0.03 & 0.37 & 0.03 & 0.44 & -0.01 & 0.71 & -0.004 & 0.92 & -0.04 & 0.27 \\
\hline Reading comprehension & 0.04 & 0.19 & 0.01 & 0.75 & 0.02 & 0.60 & -0.01 & 0.67 & -0.03 & 0.34 & -0.06 & 0.09 \\
\hline Mathematics & 0.02 & 0.48 & 0.02 & 0.61 & 0.03 & 0.32 & -0.001 & 0.97 & 0.006 & 0.86 & -0.03 & 0.32 \\
\hline
\end{tabular}

364 PGS=polygenic scores 